\title{
Aromatase expression and outcomes in the P024 neoadjuvant endocrine therapy trial
}

\author{
Matthew J. Ellis $\cdot$ William R. Miller $\cdot$ Yu Tao \\ Dean B. Evans · Hilary A. Chaudri Ross · \\ Yasuhiro Miki · Takashi Suzuki · Hironobu Sasano
}

Received: 7 August 2008/Accepted: 7 August 2008/Published online: 22 October 2008

(C) Springer Science+Business Media, LLC. 2008

\begin{abstract}
Background Expression of aromatase by malignant breast epithelial cells and/or the surrounding stroma implies local estrogen production that could influence the outcome of endocrine therapy for breast cancer. Methods A validated immunohistochemical assay for aromatase was applied to samples from the P024 neoadjuvant
\end{abstract}

M. J. Ellis $(\bowtie) \cdot$ Y. Tao

Siteman Cancer Center, Washington University School of Medicine, 660 South Euclid Ave, St. Louis, MO 63119, USA

e-mail: mellis@dom.wustl.edu

Y. Tao

e-mail: ytao@im.wustl.edu

\section{W. R. Miller}

Edinburgh Breast Unit, Edinburgh University, Edinburgh,

Scotland, UK

e-mail: w.r.miller@ed.ac.uk

D. B. Evans

Novartis Institutes for BioMedical Research, Basel, Switzerland

e-mail: dean.evans@novartis.com

H. A. Chaudri Ross

Novartis Pharma AG, Basel, Switzerland

e-mail: hilary_anne.chaudri@novartis.com

Y. Miki · T. Suzuki · H. Sasano

Department of Pathology, Tohoku University School

of Medicine, Sendai, Japan

e-mail: myasuhiro@patholo2.med.tohoku.ac.jp

T. Suzuki

e-mail: stakashi@patholo2.med.tohoku.ac.jp

H. Sasano

e-mail: hsasano@patholo2.med.tohoku.ac.jp endocrine therapy trial that compared tamoxifen and letrozole. The presence of aromatase expression by tumor or stromal cells was correlated with tumor response, treatment induced changes in proliferation index (Ki67), relapse-free survival (RFS) and breast cancer-specific survival (BCSS). Results Tumor and stromal aromatase expression were highly correlated $(P=0.0001)$. Tumor cell aromatase, as a semi-continuous score, also correlated with smaller tumor size at presentation $(P=0.01)$ higher baseline ER Allred score $(P=0.006)$ and lower Ki67 levels $(P=0.003)$. There was no significant relationship with clinical response or treatment-induced changes in Ki67. However, in a Cox multivariable model that incorporated a post-treatment tumor profile (pathological T stage, $\mathrm{N}$ stage, Ki67 and ER status of the surgical specimen), the presence of tumor aromatase expression at baseline sample remained a favorable independent prognostic biomarker for both RFS $(P=0.01$, HR $2.3,95 \%$ CI $1.2-4.6$ for absent expression $)$ and BCSS $(P=0.008$, HR 3.76, 95\% CI 1.4-10.0). Conclusions Autocrine estrogen synthesis may be most characteristic of smaller, more indolent and ER-rich breast cancers with lower baseline growth rates. However, response to endocrine treatment may not depend on whether the estrogenic stimulus has a local versus systemic source.

Keywords Aromatase - Letrozole - Tamoxifen · Neoadjuvant endocrine therapy

\section{Introduction}

After the menopause, estrogen continues to be synthesized through peripheral conversion of androgenic precursors to estrone and estradiol by the CYP P450 enzyme aromatase (CYP19). Since this enzyme is widely expressed, sources 
of estrogen for breast cancers can therefore be through the circulation (endocrine), from within the breast stroma (paracrine) or through synthesis by the tumor cell (autocrine) $[1,2]$. Intra-tumoral estrogen production has been directly demonstrated by measuring the conversion of radio-labeled androgen to estrogen in breast cancer biopsy material [1-3]. However correlations between biochemical measurements of intra-tumoral estrogen synthesis and clinical outcomes have not been firmly established, largely because in vivo assays of aromatase activity are difficult to execute in a large numbers of patients [4-6]. As alternative approaches, aromatase immunohistochemistry (IHC) and measurements of aromatase mRNA levels have been explored [7, 8]. However, most investigators have not validated their IHC assays against the "gold standard" of a biochemical assay for intra-tumoral aromatase activity. Our group has recently developed and characterized a monoclonal antibody against aromatase. The antibody has been utilized in IHC studies which demonstrated positive correlations between aromatase IHC scores and intra-tumoral aromatase activity [9] and aromatase mRNA expression measurements [10] in breast cancer specimens.

In this investigation we applied the aromatase IHC assay to formalin-fixed paraffin-embedded biopsy samples accrued from patients enrolled onto the P024 neoadjuvant endocrine therapy study, a Phase III double blind randomized trial that compared four months neoadjuvant tamoxifen with an equivalent period of letrozole treatment [11-13]. The design of this study provided a valuable opportunity to evaluate simultaneously the relationship between tumor aromatase expression and response to neoadjuvant endocrine therapy as well as the long-term outcomes for patients receiving adjuvant tamoxifen treatment.

\section{Methods}

Study population and tumor bank

The P024 protocol compared four months neoadjuvant letrozole with tamoxifen in post-menopausal women with clinical stage II and III hormone receptor positive (classified as at least $10 \%$ nuclear staining for ER and/or PgR) breast cancers that were ineligible for breast conservative surgery [11]. The tumor bank characteristics, ER and Ki67 measurements have been described previously [12, 13]. Tumor grade, tumor histological subtype, pathological staging information and long-term outcomes were collated from case report forms. The long-term outcomes and the development of the preoperative endocrine prognostic index (PEPI) based on pathological stage, and the ER status and
Ki67 expression level of the surgical specimen has also been published [14].

Aromatase immunohistochemistry

The aromatase monoclonal antibody \#677 was raised against native recombinant human aromatase protein. Details of its characterization and utilization for IHC have been previously reported [9]. Tissue sections were immunostained by a biotin-streptavidin method using a Histofine kit (Nichirei Co. Ltd., Tokyo, Japan). The antigen-antibody complex was visualized with $3.3^{\prime}$-diaminobenzidine solution (DAB) and counterstained with hematoxylin. Evaluation of aromatase IHC was performed by assessing the approximate percentage of cells staining (proportion score) and classifying the level into four groups: $0=<1 \%, 1=$ $1-25 \%, 2=26-50 \%$, and $3=>50 \%$ immuno-positive cells. The relative intensity of aromatase immune-positive cells was classified as follows: $0=$ no immunoreactivity, $1=$ weak, 2 = moderate and $3=$ intense immunoreactivity. When aromatase immunoreactivity was evaluated as a semi-continuous variable, a total score was applied that was composed of the proportion score + relative immunointensity score (SIP score). For contingency table analysis, aromatase staining was classified as any staining present versus absent staining. Immunohistochemical staining patterns of normal ducts, stromal cells, adipose cells and carcinoma cells were evaluated separately.

\section{Statistics}

All $P$ values reported were two sided; $P \leq 0.05$ were considered to be statistically significant. There was no adjustment for multiple testing. The median and interquartile range of the aromatase SIP score was calculated to show the distribution of scores. Kendall's rank correlation coefficients were used to assess relationship between aromatase SIP values and Ki67, ER, and tumor size since aromatase SIP values were ordinal variables and not normal distributed. Fisher's exact and Chi squared tests were used to define associations between aromatase expression status and clinical and cell cycle responses. The nonparametric Mann-Whitney test was applied to compare differences in Ki67 changes between aromatase expression positive and aromatase expression negative tumors. The 95\% confidence interval of the geometric Ki67 mean was calculated to show the size of effects in pair-wise comparisons. Relapse-free survival (RFS) was defined as the interval between randomization and the earliest subsequent breast cancer event (all local or systemic recurrences, there were no new breast primaries recorded in this data set). Breast cancer-specific survival (BCSS) was defined as the 
interval between randomization and the date of death after breast cancer relapse. For univariable analysis, survival curves were estimated by the Kaplan-Meier product-limit method, with a two-sided log-rank to assess statistically significant differences. We subsequently applied a multivariate Cox proportional hazards regression model to evaluate the independent prognostic relevance of aromatase expression within the context of other independently prognostic variables that were obtained upon analysis of the surgical specimen obtained after completion of neoadjuvant endocrine therapy: i.e. pathological tumor size, lymph node status, ER and Ki67 levels [14]. The REMARK analysis for the multivariable analysis has also been reported [14]. All statistical analyses were performed using SAS 9.1.2 (SAS Institute Inc., Cary NC USA).

\section{Results}

Aromatase expression and correlation with baseline pathological and clinical variables

Initially four cellular components were scored for aromatase expression (fibroblast cells, adipose cells, benign breast duct cells and invasive cancer cells). However benign ducts and adipose tissue were very inconsistently present in the slides available. Thus, only stromal cell scores and invasive cancer cell scores could be adequately studied in terms of correlations with clinical parameters. Ultimately aromatase analysis was conducted on 197 cases in which central analysis confirmed ER+ status and 23 cases in which the ER status was known to be ER negative in the central laboratory (with a cut point of Allred score of 0 or 2 as the definition of negative). Of these $197 \mathrm{ER}+$ cases, 192 (96 on letrozole, 96 on tamoxifen) had sufficient tumor cells on specimens to qualify for the analysis presented in this report. Aromatase expression SIP score in the stomal cell and tumor cell compartments were highly correlated (Kendall's Tau 0.46, $P=0.0001$, Fig. 1a) Tumor cell aromatase SIP score was positively correlated with ER levels as a continuous score (Kendall's Tau $P=0.006$, Fig. 1b), however there was no significant correlation with progesterone receptor $(\mathrm{PgR})$ level (data not shown). Finally the aromatase SIP score in the cancer compartment was inversely associated with Ki67 level (Kendall's Tau $P=0.003$ Fig. 1c). To examine correlations between aromatase expression and dichotomized clinical variables the aromatase staining score was reduced to simple present or absent categories. Of the variables examined, both stroma and tumor epithelial aromatase expression were associated with smaller clinical tumor size at baseline and ER positive status as a dichotomous variable (Allred 0-2 vs. Allred 3-8) but aromatase status

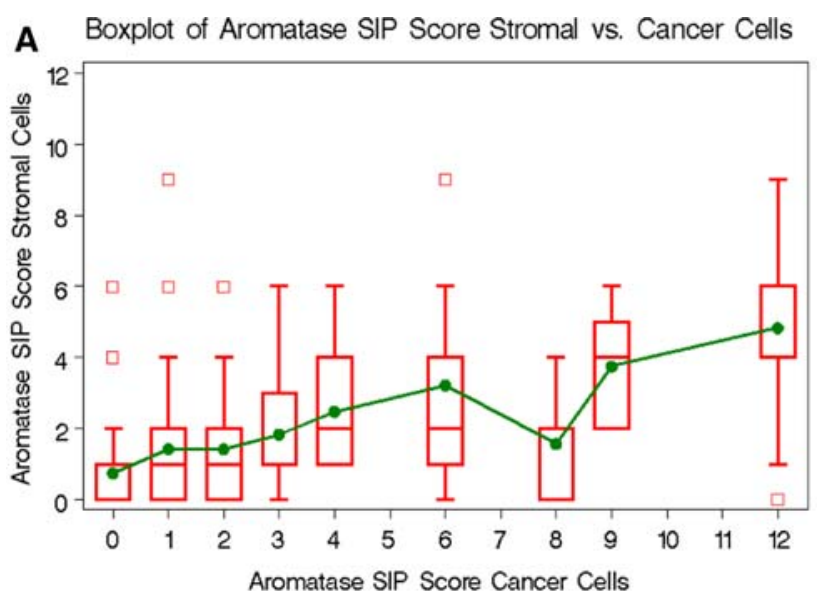

B Boxplot of ER Allred score vs. Aromatase SIP Score at Baseline
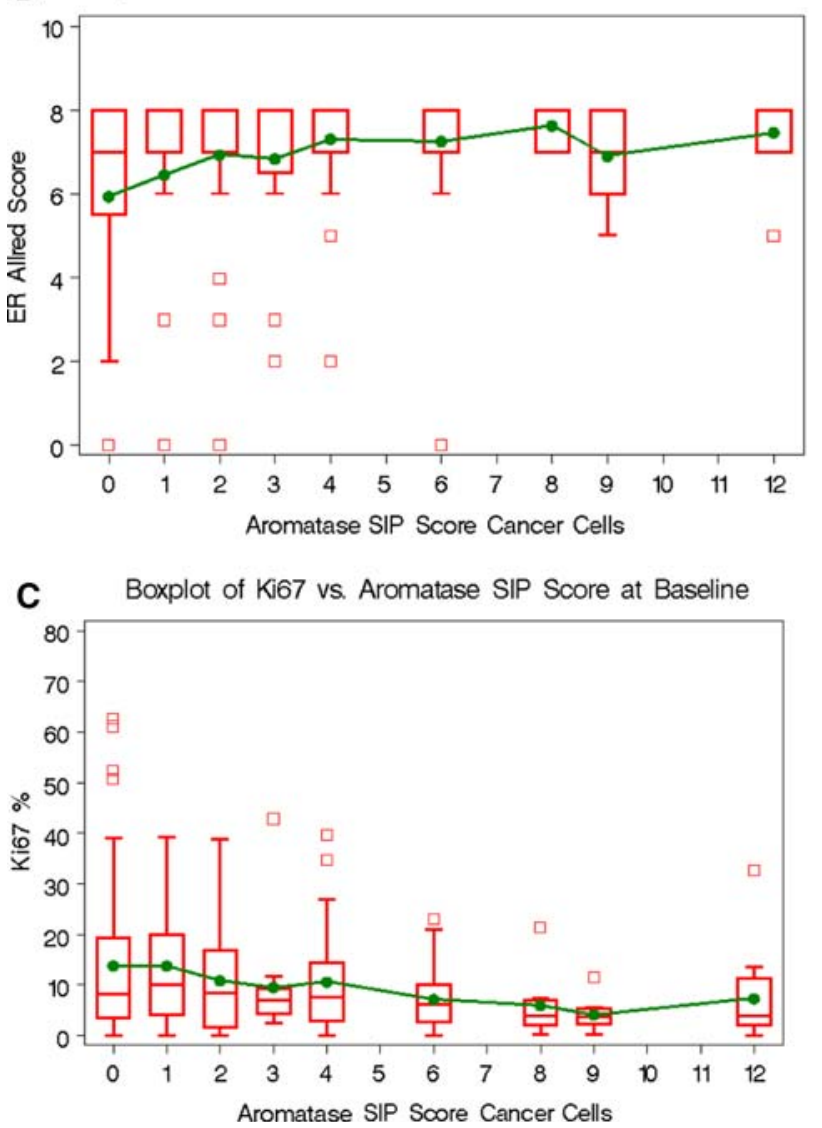

Fig. 1 Correlations between the site of aromatase expression, ER and Ki67 as semi-continuous variables. Box plots comparing the distributions of aromatase SIP scores in stromal cells and cancer cells (a), aromatase SIP scores in cancer cells and ER Allred scores (b) and Ki67 percentage and aromatase SIP scores in cancer cells (c) at baseline. The large boxes stretch from the 25 th to 75 th percentile, the lines crossing the boxes are medians, the dots are means and the small boxes are outliers.

(present vs. absent) did not interact with the other factors examined (patient age, tumor grade, lymph node status, PgR and HER2 status) (Table 1). 
Table 1 Patients and tumors characteristics by location of aromatase protein expression status at baseline a HER2 IHC with fluorescence in situ hybridization confirmation and IHC for ER and PgR were performed as previously described [12]

b Aromatase protein expression considered positive if any aromatase IHC staining was present

${ }^{c}$ For age and clinical tumor size the student's $t$ test was used to compare the aromatase positive and negative groups. For binary variables the $X^{2}$ test was applied with Fisher's exact test if a count in any cell was less than 5

\begin{tabular}{|c|c|c|c|c|}
\hline \multirow[t]{3}{*}{ Characteristics } & \multicolumn{4}{|c|}{ Aromatase protein expression $[n(\%)]^{\mathrm{b}}$} \\
\hline & \multicolumn{2}{|c|}{ Tumor epithelial aromatase } & \multicolumn{2}{|c|}{ Stromal aromatase } \\
\hline & Negative & Positive & Negative & Positive \\
\hline \multicolumn{5}{|l|}{ Treatment } \\
\hline Tamoxifen & $24(53 \%)$ & $72(49 \%)$ & $29(54)$ & $63(48)$ \\
\hline Letrozole & $21(47 \%)$ & $75(51 \%)$ & $25(46)$ & $69(52)$ \\
\hline$P$-value & 0.6102 & & 0.4605 & \\
\hline Age (year) & 66.8 & 67.6 & 67.6 & 67 \\
\hline$P$-value ${ }^{\mathrm{c}}$ & 0.6214 & & 0.6969 & \\
\hline Clinical tumor size $(\mathrm{cm})$ & 5.7 & 4.8 & 5.5 & 4.7 \\
\hline$P$-value ${ }^{\mathrm{c}}$ & 0.0144 & & 0.0398 & \\
\hline \multicolumn{5}{|l|}{ Pre treatment grade } \\
\hline I & $4(10 \%)$ & $16(13 \%)$ & $4(8 \%)$ & $16(15 \%)$ \\
\hline $\mathrm{II} / \mathrm{III}$ & $38(90 \%)$ & $103(87 \%)$ & $47(92 \%)$ & $90(85 \%)$ \\
\hline$P$-value & 0.5971 & & 0.3064 & \\
\hline \multicolumn{5}{|l|}{ Pathological tumor size } \\
\hline$\leq 20 \mathrm{~mm}$ & $11(27 \%)$ & $44(32 \%)$ & $11(27 \%)$ & $44(32 \%)$ \\
\hline$>20 \mathrm{~mm}$ & $30(73 \%)$ & $95(68 \%)$ & $30(73 \%)$ & $95(68 \%)$ \\
\hline$P$-value & 0.5567 & & 0.5567 & \\
\hline \multicolumn{5}{|l|}{ Pathological node status } \\
\hline Negative & $16(41 \%)$ & $55(43 \%)$ & $21(47 \%)$ & $50(43 \%)$ \\
\hline Positive & $23(59 \%)$ & $72(57 \%)$ & $24(53 \%)$ & $65(57 \%)$ \\
\hline$P$-value & 0.8017 & & 0.7160 & \\
\hline \multicolumn{5}{|l|}{ HER2 status $^{\mathrm{a}}$} \\
\hline Negative & $40(91 \%)$ & $140(95 \%)$ & $50(93 \%)$ & $125(95 \%)$ \\
\hline Positive & $4(9 \%)$ & $7(5 \%)$ & $4(7 \%)$ & $6(5 \%)$ \\
\hline$P$-value & 0.2806 & & 0.4810 & \\
\hline \multicolumn{5}{|l|}{ ER status ${ }^{c}$} \\
\hline Negative & $11(20 \%)$ & $12(8 \%)$ & $14(20 \%)$ & $9(6 \%)$ \\
\hline Positive & $44(80 \%)$ & $148(93 \%)$ & $55(80 \%)$ & $131(94 \%)$ \\
\hline$P$-value & 0.0098 & & 0.0027 & \\
\hline \multicolumn{5}{|l|}{ PgR status ${ }^{c}$} \\
\hline Negative & $16(36 \%)$ & $48(33 \%)$ & $20(37 \%)$ & $43(33 \%)$ \\
\hline Positive & $28(64 \%)$ & $98(67 \%)$ & $34(63 \%)$ & $87(67 \%)$ \\
\hline$P$-value & 0.6688 & & 0.6072 & \\
\hline
\end{tabular}

Aromatase expression and clinical or radiological response to neoadjuvant letrozole or tamoxifen

A series of contingency tables were examined to identify interactions between aromatase expression status and response (Table 2). In the P024 study, response was recorded according to clinical measurements, ultrasound and mammography. There was no evidence of interactions with any of the response definitions, whether the stroma or the tumor cell aromatase status was examined as the interacting factor or whether letrozole or tamoxifen treated cases were considered separately. Consistent with a lack of an influence on endocrine therapy responsiveness, there was no interaction with treatment-induced changes in Ki67 or absolute post-treatment Ki67 levels in either tamoxifen or letrozole-treated tumor samples (Table 3).

Aromatase expression and relapse-free survival and breast cancer-specific survival

Although there was no association with neoadjuvant response or Ki67 changes, the baseline interactions between aromatase expression, higher ER levels and lower Ki67 levels suggested the possibility that aromatase expression could be a favorable prognostic biomarker for patients undergoing adjuvant endocrine therapy. We therefore examined the impact of aromatase expression on RFS and BCSS (Fig. 2). Tumor aromatase expression was 
Table 2 Analysis of clinical, mammogram and ultrasound response data according to aromatase protein expression status in tamoxifen or letrozole treated patients

\begin{tabular}{llll}
\hline Responses & \multicolumn{2}{l}{ Aromatase protein expression $[n(\%)]$} \\
\cline { 2 - 3 } & Tumor epithelial aromatase & & Stromal aromatase \\
\cline { 2 - 4 } & Negative $\quad$ Positive & & Negative $\quad$ Positive \\
\hline
\end{tabular}

Clinical response ${ }^{\mathrm{a}}$

Letrozole only

$\begin{array}{lllll}\text { No } & 6(29 \%) & 24(32 \%) & 11(44 \%) & 18(26) \\ \text { Yes } & 15(71 \%) & 51(68 \%) & 14(56 \%) & 51(74) \\ P \text {-value } & 0.7657 & & 0.0984 & \end{array}$

Tamoxifen only

$\begin{array}{lllll}\text { No } & 12(50 \%) & 37(51 \%) & 13(45 \%) & 34(54 \%) \\ \text { Yes } & 12(50 \%) & 35(49 \%) & 16(55 \%) & 29(46 \%) \\ P \text {-value } & 0.9067 & & 0.4177 & \end{array}$

Fused

$\begin{array}{lllll}\text { No } & 18(40 \%) & 61(41 \%) & 24(44 \%) & 52(39 \%) \\ \text { Yes } & 27(60 \%) & 86(59 \%) & 30(56 \%) & 80(61 \%) \\ P \text {-value } & 0.8587 & & 0.5259 & \end{array}$

Mammo response ${ }^{\mathrm{a}}$

Letrozole only

$\begin{array}{lllll}\text { No } & 13(62 \%) & 47(63 \%) & 17(68 \%) & 42(61 \%) \\ \text { Yes } & 8(38 \%) & 28(37 \%) & 8(32 \%) & 27(39 \%) \\ P \text {-value } & 0.9494 & & 0.5297 & \end{array}$

Tamoxifen only

$\begin{array}{lllll}\text { No } & 19(79 \%) & 54(75 \%) & 23(79 \%) & 48(76 \%) \\ \text { Yes } & 5(21 \%) & 18(25 \%) & 6(21 \%) & 15(24 \%) \\ P \text {-value } & 0.6803 & & 0.7418 & \end{array}$

Fused

$\begin{array}{lllll}\text { No } & 32(71 \%) & 101(69 \%) & 40(74 \%) & 90(68 \%) \\ \text { Yes } & 13(29 \%) & 46(31 \%) & 14(26 \%) & 42(32 \%) \\ P \text {-value } & 0.7604 & & 0.4278 & \end{array}$

Ultrasound response ${ }^{\mathrm{a}}$

Letrozole only

$\begin{array}{lllll}\text { No } & 12(67 \%) & 39(56 \%) & 16(73 \%) & 34(52 \%) \\ \text { Yes } & 6(33 \%) & 31(44 \%) & 6(27 \%) & 31(48 \%) \\ P \text {-value } & 0.4038 & & 0.0959 & \end{array}$

Tamoxifen only

$\begin{array}{lllll}\text { No } & 13(68 \%) & 44(64 \%) & 15(60 \%) & 41(69 \%) \\ \text { Yes } & 6(32 \%) & 25(36 \%) & 10(60 \%) & 18(31 \%) \\ P \text {-value } & 0.7085 & & 0.4016 & \\ \text { No } & 25(68 \%) & 83(60 \%) & 31(66 \%) & 75(60 \%) \\ \text { Yes } & 12(32 \%) & 56(40 \%) & 16(34 \%) & 49(40 \%) \\ P \text {-value } & 0.3845 & & 0.5116 & \end{array}$

Response rate refers to the percentage of patients with a complete or partial response

${ }^{\text {a }}$ Response definitions by WHO criteria have been previously reported [11]. The $X^{2}$ test was applied with Fisher's exact test if a count in any cell was less than 5 confirmed to have a modest association with a more favorable disease course, with fewer relapse events over time and a significant univariable $\log$ rank test $P=0.04$ (Fig. 2a) and more prolonged breast cancer survival (Fig. 2b $P=0.01$ ). To determine the independence of baseline aromatase expression as a prognostic marker in our established multivariable models based on the posttreatment surgical sample, the baseline aromatase status was analyzed in the context of the preoperative endocrine relapse index (PEPI) (Table 4) [14]. In the PEPI model pathologic tumor size (T1/2 vs. T3/4), pathological nodal status (negative vs. positive), Ki67 per natural log interval and ER status post therapy (Allred 0-2 vs. Allred 3-8) have been found to be independent factors for RFS and BCSS [14]. When tumor aromatase status was entered into a multivariable Cox model containing these four factors, the presence of aromatase expression in the baseline specimen behaved as an independent favorable prognostic biomarker for both RFS $(P=0.01$, HR $2.395 \% 1.2-4.6$ for absent expression) (Table 4A) and BCSS $(P=0.008$, HR 3.76 95\% CI 1.4-10.0 for absent expression) (Table 4B).

\section{Discussion}

The clinical significance of intra-tumoral estrogen production has been debated ever since the phenomenon was first documented by Miller et al., in 1974 [15] through the detection of the conversion of radio-labeled androgen to estradiol within breast cancers in vitro. This potential exists in about $60-70 \%$ of breast cancers [1-3]. Subsequently infusion studies with radioactive androgens showed that estrogen biosynthesis occurred in situ within the breast [16, 17] and the presence of mRNA for aromatase, the key enzyme in estrogen production, was also demonstrated in breast cancers and adipose tissue [8]. Because aromatase is the last step in the biosynthetic pathway for estradiol, the enzyme has become a critical target for pharmacological inhibitors that achieve endocrine deprivation for postmenopausal patients requiring endocrine treatment for ER + breast cancer. Consequently third-generation aromatase inhibitors have evolved as the new standard of care for breast cancer treatment for all stages of the disease. It was therefore logical to address the possibility that the presence of aromatase within breast cancers is associated with a particular requirement for estrogen for growth and therefore whether aromatase expressing tumors are more likely to respond to endocrine therapy in general, and to aromatase inhibitors in particular.

The number of studies examining these relationships is few, have utilized small numbers of tumors and come to limited (often conflicting) conclusions [4, 18, 19]. The 
Table 3 Paired Ki67 data before and after letrozole/tamoxifen therapy according to aromatase protein expression status in breast cancer cells/ stromal cells

\begin{tabular}{|c|c|c|c|c|}
\hline \multirow[t]{3}{*}{ Ki67 [Geometric mean $(95 \% \mathrm{CI})]^{\mathrm{a}}$} & \multicolumn{4}{|c|}{ Aromatase protein expression } \\
\hline & \multicolumn{2}{|l|}{ Cancer cells } & \multicolumn{2}{|l|}{ Stromal cells } \\
\hline & Negative & Positive & Negative & Positive \\
\hline \multicolumn{5}{|l|}{ Letrozole only } \\
\hline Pre & $5.54(2.54-12.08)$ & $3.56(2.47-5.14)$ & $3.64(1.70-7.82)$ & $3.92(2.69-5.72)$ \\
\hline Post & $0.70(0.33-1.49)$ & $0.49(0.31-0.75)$ & $0.88(0.38-2.04)$ & $0.44(0.29-0.68)$ \\
\hline$P$-value ${ }^{\mathrm{b}}$ & 0.0037 & 0.0001 & 0.0083 & 0.0001 \\
\hline \multicolumn{5}{|l|}{ Tamoxifen only } \\
\hline Pre & $5.97(3.17-11.24)$ & $5.63(4.18-7.58)$ & $7.75(4.46-13.47)$ & $4.67(3.39-6.44)$ \\
\hline Post & $1.72(0.75-3.97)$ & $1.36(0.88-2.09)$ & $1.61(0.76-3.39)$ & $1.23(0.77-1.95)$ \\
\hline$P$-value ${ }^{\mathrm{b}}$ & 0.0117 & 0.0001 & 0.0007 & 0.0001 \\
\hline
\end{tabular}

a $95 \%$ CI: confidence Interval

${ }^{\mathrm{b}}$ Wilcoxon signed rank test was used to compare paired Ki67 data within each group defined by aromatase expression status

major reason for this is that the low abundance of aromatase in the breast requires sophisticated, time-consuming and labor intensive methodology and relatively large amounts of fresh tissue. This has precluded routine use in large clinical trials. However the availability of an antibody which can specifically detect aromatase in fixed archival breast cancers has changed this. We can now report results on the presence (and semi-quantitative levels) of aromatase in tumor material obtained from a randomized trial of neoadjuvant endocrine therapy (P024).

Before discussing the findings it is worth considering methodological issues and potential limitations of the study. Firstly, IHC estimation of protein provides no information on activity and protein may be present that is deactivated or inhibited [20]. This certainly will be the case in patients treated with aromatase inhibitors. For this reason we have excluded outcome correlations with aromatase status in "on treatment" samples because we have not validated relationships between aromatase activity and expression in the presence of an endocrine agent. Secondly, because aromatase is present in different compartments of the breast (and at different levels) complete assessment requires quantification of multiple tissue types and an estimate of the relative amounts of each compartment. In this study, to simplify these confounders, we have not used assessments in adipose and benign tissue of tissue sections which were generally low in staining score and proportion. Aromatase scores were highest in the malignant and stromal compartments of breast cancers. However, these were highly related in breast cancers suggesting a field effect of trophic factors regulating aromatase. We have therefore restricted our correlations to the status of the cancer cells which were reliably present in all the samples eligible for analysis and therefore more consistent to score.
In terms of demographics we have combined the two arms of the P024 trial for long term outcome analysis, not discriminating between patients subsequently treated with tamoxifen or letrozole since all patients received tamoxifen as adjuvant therapy. This has formed a database which represents the largest published series of breast cancers assessed by aromatase IHC. The results show that tumor aromatase was positively and significantly related to smaller tumor size and ER level/status. These findings would be consistent with data published by members of the group on aromatase activity [4] but not with others using IHC with a different antibody [7]. A significant inverse correlation was observed with the proliferation marker, Ki67. To the best of our knowledge there have been no other published studies relating tumor aromatase to proliferation.

In terms of endocrine responsiveness, no significant association was detected between tumor aromatase and clinical response to either letrozole or tamoxifen. While positive correlations have been reported between the presence of in vitro and in vivo aromatase activity and response to aromatase inhibitors, these relationships were not strong and were observed in advanced disease, not in the neoadjuvant setting [18, 19] Other studies on response to tamoxifen have been negative. Thus, the response to endocrine therapy does not appear to be strongly modulated by whether the source of estrogen is autocrine or endocrine.

Despite a failure to observe significant relationships between aromatase expression and clinical or biomarker response to treatment in the neoadjuvant phase of the study, significant associations were found between the presence of tumor aromatase expression and long-term outcome following neoadjuvant treatment. Thus, tumors with positive aromatase scores had significantly greater 
Table 4 Univariate and multivariate analysis of pathological tumor size, node status, post-treatment Ki67, post-treatment ER and pre treatment aromatase status $^{\mathrm{a}}$

\begin{tabular}{|c|c|c|c|c|c|c|}
\hline \multirow[t]{3}{*}{ Factor definitions } & \multirow{3}{*}{$\begin{array}{l}\text { No. of patients } \\
\text { in each group }\end{array}$} & \multirow{3}{*}{$\begin{array}{l}\text { No. of events/ } \\
\text { No. of patients }\end{array}$} & \multicolumn{4}{|c|}{ Relapse-free survival } \\
\hline & & & \multicolumn{2}{|c|}{ Univariable analysis } & \multicolumn{2}{|c|}{ Multivariate analysis } \\
\hline & & & $\mathrm{HR}(95 \% \mathrm{CI})$ & $P$ & $\mathrm{HR}(95 \% \mathrm{CI})$ & $P$ \\
\hline $\begin{array}{l}\text { Tumor size }^{\mathrm{a}} \\
\text { (T1/2 vs. T3/4) }\end{array}$ & $138 / 33$ & $47 / 171$ & $2.7(1.4-5.0)$ & 0.002 & $2.82(1.36-5.85)$ & 0.006 \\
\hline $\begin{array}{l}\text { Node status } \\
\text { (Yes vs. No) }\end{array}$ & $90 / 69$ & $44 / 159$ & $3.9(1.8-8.4)$ & 0.0005 & $3.44(1.58-7.48)$ & 0.002 \\
\hline Ki67 level, per 2.7 fold increase ${ }^{b}$ & & $48 / 174$ & $1.4(1.2-1.6)$ & 0.0002 & $1.1(1.02-1.09)$ & 0.003 \\
\hline $\begin{array}{l}\text { ER Allred }^{\mathrm{c}} \\
(0.2 \text { vs. } 3-8)\end{array}$ & $16 / 157$ & $48 / 173$ & $2.4(1.0-5.3)$ & 0.04 & $2.74(1.1-6.67)$ & 0.03 \\
\hline $\begin{array}{l}\text { Aromatase status }{ }^{\mathrm{d}} \\
\text { (not present versus present) }\end{array}$ & $37 / 132$ & $48 / 169$ & $1.88(1.01-3.47)$ & 0.04 & $2.34(1.2-4.58)$ & 0.01 \\
\hline \multicolumn{7}{|l|}{ B } \\
\hline \multirow[t]{3}{*}{ Factor definitions } & \multirow{3}{*}{$\begin{array}{l}\text { No. of patients } \\
\text { in each } \\
\text { group }\end{array}$} & \multirow{3}{*}{$\begin{array}{l}\text { No. of events/ } \\
\text { No. of } \\
\text { patients }\end{array}$} & \multicolumn{4}{|c|}{ Breast cancer-specific survival } \\
\hline & & & \multicolumn{2}{|c|}{ Univariable analysis } & \multicolumn{2}{|l|}{ Multivariate analysis } \\
\hline & & & $\mathrm{HR}(95 \% \mathrm{CI})$ & $P$ & $\mathrm{HR}(95 \% \mathrm{CI})$ & $P$ \\
\hline $\begin{array}{l}\text { Tumor size } \\
\text { (T1/2 vs. T3/4) }\end{array}$ & $138 / 33$ & $24 / 171$ & $3.5(1.5-8.3)$ & 0.004 & $3.42(1.21-9.66)$ & 0.02 \\
\hline $\begin{array}{l}\text { Node status } \\
\text { (Yes vs. No) }\end{array}$ & $90 / 69$ & $22 / 159$ & $4.6(1.4-15.8)$ & 0.01 & $4.05(1.14-14.38)$ & 0.03 \\
\hline Ki67 level, per 2.7 fold increase & & $25 / 174$ & $1.4(1.1-1.7)$ & 0.009 & $1.05(1.0-1.11)$ & 0.06 \\
\hline $\begin{array}{l}\text { ER Allred } \\
(0.2 \text { vs. } 3-8)\end{array}$ & $16 / 157$ & $25 / 173$ & $4.3(1.6-11.7)$ & 0.005 & $7.98(2.58-24.7)$ & 0.0003 \\
\hline $\begin{array}{l}\text { Aromatase status } \\
\text { (not present versus present) }\end{array}$ & $37 / 132$ & $24 / 169$ & $2.82(1.2-6.63)$ & 0.02 & 3.76 (1.42-9.98) & 0.008 \\
\hline
\end{tabular}

${ }^{a}$ The four elements of the preoperative endocrine relapse index (PEPI) score (pathological T and N stage, surgical specimen ER and Ki67 status has been previously described [14]

b Surgical specimen Ki67 was analyzed as the natural log interval, or per 2.7 fold increase according to the original scale of percentage values [14]

c The ER analysis refers to the post-treatment values, before treatment all the tumors in this data set were ER positive. In the PEPI model, an Allred cut off of 0 or 2 is used to define ER negative

${ }^{d}$ The aromatase expression status was defined as present or positive if any positive staining presented in invasive breast cancer cells. Table $4 \mathrm{~A}$ and B shows the RFS data and BCSS data, respectively

RFS and BCSS. It is not possible to ascertain whether this is directly caused by increased sensitivity to endocrine therapy in the adjuvant setting. However, the lack of association of response in the neoadjuvant situation would not be compatible with this. Furthermore the positive correlations with small clinical size and ER status levels (favorable prognostic biomarkers) and the inverse correlation with $\mathrm{Ki} 67$ (a poor prognosis biomarker) suggest that aromatase positive tumors may be inherently less aggressive. This is supported by data from multivariable analyses in which tumor aromatase scores predicted for long-term outcome independently of other factors that have been shown to be predictive for outcome in the post neoadjuvant endocrine therapy setting. This finding also implies that the most accurate models for the prediction of outcomes for patients with ER+ disease may combine baseline prognostic biomarker analysis, in combination with the "ontreatment" predictive biomarker analysis derived from an analysis of the tumor after several months of endocrine treatment [14].

It is therefore suggested that routine IHC measurements of aromatase in breast cancer will not generally aid 

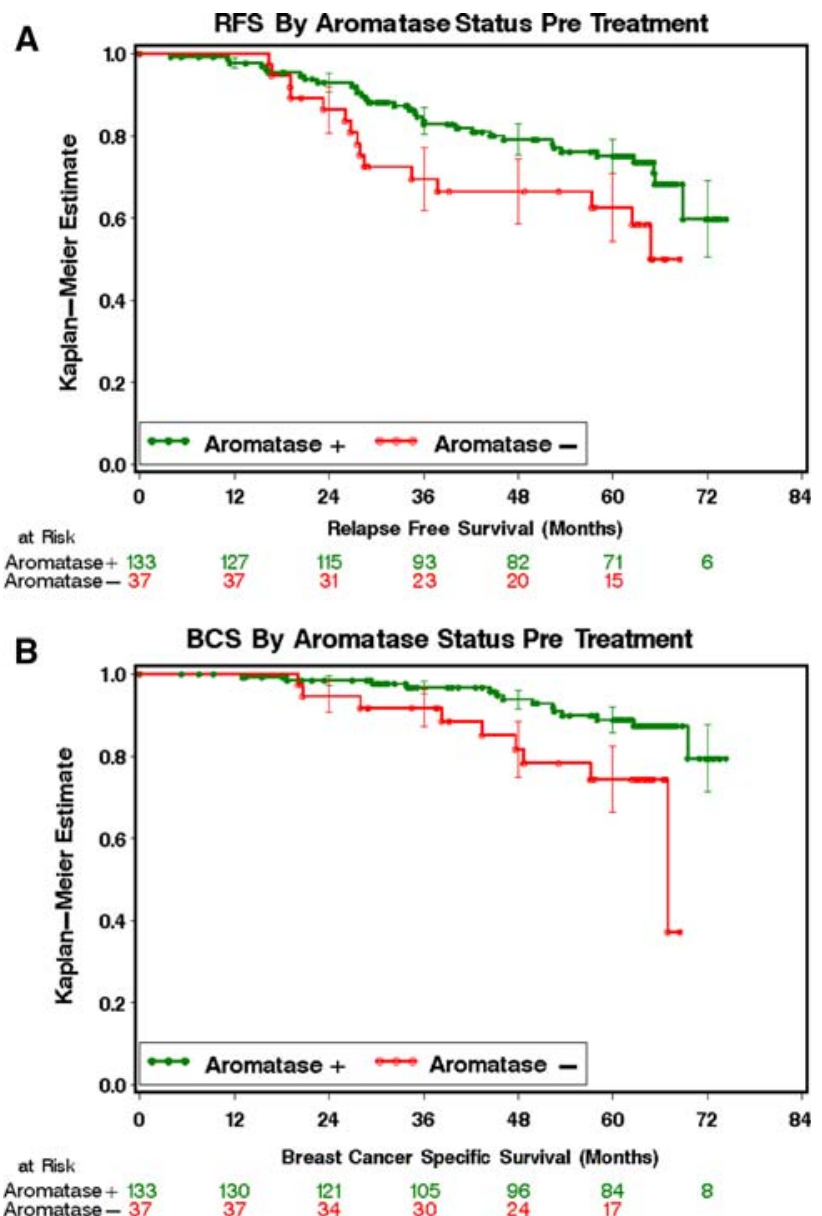

Fig. 2 Kaplan-Meier curves for relapse-free and breast cancerspecific survival by aromatase protein expression status in cancer cells. (a) Relapse-free survival (RFS) for patients with aromatase protein expression positive (green or upper curve) versus negative (red or lower curve) in cancer cells; (b) Breast cancer-specific survival (BCSS) for patients with aromatase protein expression positive (green or upper curve) versus negative (red or lower curve) in cancer cells; Censorship observations are marked with open circles; log rank tests were used to estimate the difference between KaplanMeier curves for RFS and BCSS. 95\% confidence intervals are provided on each curve

prediction of neoadjuvant response to endocrine therapy, but may help identify ER positive tumors with favorable long-term outcomes.

\section{References}

1. Reed MJ (1994) The role of aromatase in breast tumors. Breast Cancer Res Treat 30(1):7-17. doi:10.1007/BF00682737

2. Miller WR (2006) Aromatase inhibitors and breast cancer. Minerva Endocrinol 31(1):27-46

3. Miller WR, Hawkins RA, Forrest AP (1982) Significance of aromatase activity in human breast cancer. Cancer Res $42(8$ Suppl):3365s-3368s

4. Miller WR, Anderson TJ, Jack WJ (1990) Relationship between tumour aromatase activity, tumour characteristics and response to therapy. J Steroid Biochem Mol Biol 37(6):1055-1059. doi: 10.1016/0960-0760(90)90465-W

5. Miller WR (1991) Aromatase activity in breast tissue. J Steroid Biochem Mol Biol 39(5B):783-790. doi:10.1016/0960-0760(91) 90026-2

6. Lipton A, Santen RJ, Santner SJ, Harvey HA, Sanders SI, Matthews YL (1992) Prognostic value of breast cancer aromatase. Cancer 70(7):1951-1955. doi :10.1002/1097-0142(19921001)70: 7<1951::AID-CNCR2820700723>3.0.CO;2-\#

7. Esteban JM, Warsi Z, Haniu M, Hall P, Shively JE, Chen S (1992) Detection of intratumoral aromatase in breast carcinomas. An immunohistochemical study with clinicopathologic correlation. Am J Pathol 140(2):337-343

8. Miki Y, Suzuki T, Sasano H (2007) Controversies of aromatase localization in human breast cancer-stromal versus parenchymal cells. J Steroid Biochem Mol Biol 106(1-5):97-101

9. Sasano H, Anderson TJ, Silverberg SG, Santen RJ, Conway M, Edwards DP, Krause A, Bhatnagar AS, Evans DB, Miller WR (2005) The validation of new aromatase monoclonal antibodies for immunohistochemistry-a correlation with biochemical activities in 46 cases of breast cancer. J Steroid Biochem Mol Biol 95(1-5):35-39

10. Miki Y, Suzuki T, Tazawa C, Yamaguchi Y, Kitada K, Honma S et al (2007) Aromatase localization in human breast cancer tissues: possible interactions between intratumoral stromal and parenchymal cells. Cancer Res 67(8):3945-3954. doi:10.1158/ 0008-5472.CAN-06-3105

11. Eiermann W, Paepke S, Appfelstaedt J, Llombart-Cussac A, Eremin J, Vinholes J et al (2001) Preoperative treatment of postmenopausal breast cancer patients with letrozole: a randomized double-blind multicenter study. Ann Oncol 12(11):15271532. doi:10.1023/A:1013128213451

12. Ellis MJ, Coop A, Singh B, Mauriac L, Llombert-Cussac A, Janicke $F$ et al (2001) Letrozole is more effective neoadjuvant endocrine therapy than tamoxifen for ErbB-1- and/or ErbB-2positive, estrogen receptor-positive primary breast cancer: evidence from a phase III randomized trial. J Clin Oncol 19(18): 3808-3816

13. Ellis MJ, Coop A, Singh B, Tao Y, Llombart-Cussac A, Janicke F et al (2003) Letrozole inhibits tumor proliferation more effectively than tamoxifen independent of HER $1 / 2$ expression status. Cancer Res 63(19):6523-6531

14. Ellis MJ, Tao Y, Luo J, A'Hern R, Evans DB, Bhatnagar AJ et al (2008) Outcome prediction for estrogen receptor-positive breast cancer based on post neoadjuvant endocrine therapy tumor characteristics. J Natl Cancer Inst 100(19): (in press)

15. Miller WR, Forrest AP (1974) Oestradiol synthesis by a human breast carcinoma. Lancet 2(7885):866-868. doi:10.1016/S01406736(74)91202-1

16. Reed MJ, Aherne GW, Ghilchik MW, Patel S, Chakraborty J (1991) Concentrations of oestrone and 4-hydroxyandrostenedione in malignant and normal breast tissues. Int J Cancer 49(4):562565. doi:10.1002/ijc. 2910490415

17. Miller WR (1997) Uptake and synthesis of steroid hormones by the breast. Endocr Relat Cancer 4:307-311

18. Bezwoda WR, Mansoor N, Dansey R, Esser JD (1987) Aromatisation of androstenedione by human breast cancer tissue: correlation with hormone receptor activity and possible biologic significance. Oncology 44(1):30-33

19. Miller WR, Anderson TJ, Evans DB, Krause A, Hampton G, Dixon JM (2003) An integrated view of aromatase and its inhibition. J Steroid Biochem Mol Biol 86(3-5):413-421. doi: 10.1016/S0960-0760(03)00352-2

20. Miller WR, Dixon JM (2002) Endocrine and clinical endpoints of exemestane as neoadjuvant therapy. Cancer Control 9(2 Suppl): $9-15$ 